\title{
El vídeo, el cómic y el rap: una forma diferente de desbloquear al candidato del DELE
}

\author{
FLORENCIA BATTAGLIERO BOCCO \\ Taronja School \\ flobatt@alumni.uv.es \\ José Vicente FonTANa OlTRA \\ Taronja School \\ MARTA NAVARRO
}

Taronja School

Resumen: El objetivo del presente artículo es trabajar y reforzar las destrezas y competencias necesarias para aprobar el Diploma de Español como Lengua Extranjera. Para ello, se planteará una serie de recursos que buscan desbloquear al estudiante en un ambiente distendido, sobre todo los días previos al examen. La propuesta se centrará en la Prueba de Expresión e Interacción Orales, concretamente en la tarea 3 del nivel B1 y en la tarea 2 del nivel B2, que corresponden a la descripción de una fotografía. Se empleará, por una parte, un vídeo de carácter estático (donde el interlocutor no expresa movimientos corporales pronunciados) y por otra, cómics de Argentina que a su vez servirán para ampliar el léxico en la variante rioplatense. Por último, se tratará el uso del rap como herramienta para mejorar la pronunciación, entonación y el empleo del lenguaje no verbal.

Palabras claves: DELE, vídeo, cómic, rap.

Abstract: The purpose of the following essay is to train and to strengthen the skills and the competences required to pass the "Spanish as a Foreign Language Certificate". In order to do so, we will first consider various resources which aim to release students from any kind of stress by setting up a relaxed atmosphere - especially a few days before the exam. The focus of the present essay has to be linked to the Expression and Oral Interaction tests and above all to the assignments 3 (B1 level) and 2 (B2 level), and their picture descriptions. On the one hand, we will use a static video and, on the other hand, we will tackle Argentinian comics in order to expand students' knowledge of the Río de la Plata lexicon. Finally, we will deal with the use of rap as a tool to improve pronunciation, intonation, and non-verbal communication.

Key words: DELE, video, comic, rap. 


\section{Introducción}

El español es un idioma en auge, razón por la cual miles de personas de todo el mundo se presentan a los exámenes DELE. Estas pruebas, diseñadas bajo las directrices del Marco Común Europeo de Referencia (MCER), permiten obtener un título acreditativo oficial expedido por el Instituto Cervantes del nivel al que el estudiante se presenta. Así pues, se trata de uno de los títulos con más prestigio y validez actual.

En cuanto a la preparación de un examen de tal calibre, dicha situación provoca, en muchos casos, un estado de estrés en los estudiantes. Como consecuencia, estos factores impiden que los candidatos demuestren su nivel de español real.

Sumado a este inconveniente, es necesario que dominen unas competencias mínimas que, a veces, puede que no manejen bien en su propio idioma. Por ejemplo, es posible que no estén acostumbrados a organizar la información con el fin de crear y estructurar un discurso de manera coherente, que no se sientan capaces de elaborar hipótesis a partir de un estímulo mínimo, como lo es una fotografía, así como la dificultad de desenvolverse con aparente naturalidad en temas que desconocen.

Para subsanar la problemática planteada, en el presente artículo se presentarán tres recursos que se caracterizan por alejarse del formato estándar. Se trata de un vídeo, de tiras de cómic y de música rap, enmarcados en tres propuestas didácticas que pueden ser utilizadas según las necesidades del profesor, ya que son materiales flexibles y adaptables a diferentes niveles, atendiendo siempre a las características específicas de cada grupo.

Para empezar, se explicará la metodología que se basa en el empleo de un vídeo de carácter estático adjunto a un esquema orientativo, cuyo fin es trabajar la descripción de la fotografía de la Tarea 2 de la Prueba de Expresión e Interacción Oral del DELE B2 (y por extensión, la Tarea 3 de la Prueba de Expresión e Interacción Oral del DELE B1).

A continuación, sirviéndonos del mismo esquema, se desarrollará el empleo del cómic, introduciendo además el léxico y la morfología de la variedad del español de Argentina. Por último, se expondrá la utilización del estilo musical del rap, trabajando de este modo la entonación, la mejora de la pronunciación y el lenguaje no verbal; sumado al hecho de que se acerca a los estudiantes a un léxico nuevo.

\section{No saben qué decir y yo no sé qué hacer. El vídeo como herramienta de descripción}

¿Por qué se propone recurrir a un vídeo y no directamente a la fotografía? Si trabajamos el vídeo de un fragmento de discurso político (cuya duración máxima es de un minuto) en una lengua no reconocible por los aprendices, lo que se pretende es provocar una situación de «confusión controlada» en la que se afronta el bloqueo del estudiante desde una perspectiva diferente, sin dejar de trabajar los contenidos pertinentes al examen DELE. A través del visionado, ofrecemos al estudiante una vía para reconocer elementos del vídeo apoyándose en el contexto y los elementos pragmáticos, a partir de los cuales realizará hipótesis en indicativo y en subjuntivo. En cuanto a la LA (lengua para la actividad), que escojamos, siempre dependerá de las necesidades y de las características del grupo, ya que podemos optar por un vídeo que utilice un idioma perteneciente a una familia lingüística del español 
o a una que esté totalmente alejada. Por ejemplo, si nuestra intención es que lleguen a comprender algunos sintagmas, podemos, por una parte, recurrir a un vídeo ${ }^{1}$ en catalán o valenciano del que podrán inferir ciertas palabras similares a las de la lengua meta o, por otra parte, usar un vídeo que se valga de una lengua sin similitudes con la lengua madre del estudiante ni de la lengua meta, como podría ser el caso del japonés ${ }^{2}$.

Respecto al procedimiento, primero se efectúa el visionado provocando un sentimiento de confusión al invitar al futuro candidato a realizar una descripción del vídeo siguiendo las consignas que aparecen en los modelos de examen de la Tarea 2. Seguidamente, se ofrece al aprendiente un esquema que puede seguir a modo de guía descriptiva, este le permite realizar una descripción orientada y óptima.

Una vez terminada esta actividad, el estudiante suele llevar a cabo la descripción de la fotografía con más facilidad, puesto que acaba de afrontar la misma situación, pero en un contexto mucho más complicado y en el que resulta mucho más difícil aportar información o describir imágenes sin comprender el mensaje. La guía que se ha usado se aprovecha también para reforzar estructuras, pero, sobre todo, para incentivar al estudiante a formular hipótesis. Es decir, el objetivo es ofrecer recursos que eviten las largas pausas o reflexiones con las que los candidatos buscan y estructuran la información. Así pues, el esquema mencionado consta de cuatro puntos básicos que analizaremos a continuación:

\begin{tabular}{|l|l|}
\hline $\begin{array}{l}\text { 1.Identificación: ¿Qué y/o quién es? } \\
\text { Hipótesis en indicativo y subjuntivo con } \\
\text { quizás, probablemente, posiblemente, tal } \\
\text { vez etc. }\end{array}$ & $\begin{array}{l}\text { 2.Ubicación y estado: ¿Dónde y cómo } \\
\text { está? }\end{array}$ \\
\hline $\begin{array}{l}\text { Expresar estado y localización. Realizar } \\
\text { hipótesis con indicativo y subjuntivo } \\
\text { como en el punto 1. }\end{array}$ \\
$\begin{array}{l}\text { 3.Motivo: ¿Por qué? } \\
\text { Hipótesis de pasado y futuro combinando } \\
\text { compuesto. }\end{array}$ & $\begin{array}{l}\text { 4.Acción: ¿Qué ha dicho? ¿Qué está } \\
\text { pasando? } \\
\text { Trabajar el estilo indirecto }\end{array}$ \\
\hline
\end{tabular}

Tabla 1. Guía descriptiva

Después de familiarizar al estudiante con el esquema, el docente trabaja la dinámica en clase, de manera que el estudiante se familiarice con el hecho de hablar tanto de lo conocido o evidente, como de aquello que le es desconocido. Es por ello que el énfasis radica en la ordenación de la información y en la realización de hipótesis. Además, se invitará a los aprendientes a realizar hipótesis tanto en futuro como en pasado con el objetivo de revisar el mayor número de tiempos verbales posible.

1 Mónica Oltra (mayo y junio de 2016)

2 Shinzo Abe (noviembre de 2013) 
El docente puede empezar recordando a los estudiantes que la diferencia entre expresar una hipótesis en indicativo o en subjuntivo depende del grado de seguridad que se desea transmitir, y que hay palabras que pueden introducirse en ambos modos. El punto 1 y el 2 se centran en las hipótesis en presente, en cambio el punto 3 se centra en la expresión de hipótesis en pasado y en futuro, remarcando la combinación de futuro perfecto y pretérito perfecto de subjuntivo, ya que los dos expresan hipótesis en los mismos tiempos. De esta manera, conseguimos ampliar la perspectiva que tiene el estudiante sobre la lengua, alejándole del encasillamiento de tiempos verbales propios del bloqueo mencionado anteriormente. Finalizamos con el punto 4, en el que trabajaremos el estilo indirecto, y en el que el estudiante intentará deducir de qué ha hablado la persona del vídeo.

Con el desarrollo de esta actividad hemos conseguido aumentar la confianza del estudiante de español ante situaciones de apariencia compleja, ya que trabajar estos recursos puede ayudarle a expresarse mejor sobre lo desconocido, así como reforzar estructuras lingüísticas, o a practicar su fluidez, corrección y coherencia, aspectos que le ayudarán en la superación de la Tarea 2 de la parte de interacción y expresión orales del examen.

\section{El cómic como recurso creativo}

Sumado a los inconvenientes mencionados anteriormente, otro factor de riesgo al que se enfrentan muchos estudiantes es la sensación de falta de imaginación que se añade al estrés generado al pensar que en un examen cuya tarea es semilibre, solo existe una respuesta correcta. Para combatir esta situación, la abstracción que provocan los dibujos de un cómic, así como el fomento de la imaginación y la capacidad creativa, hacen de este recurso una herramienta útil para usar como calentamiento previo al trabajo con muestras reales del examen DELE tanto en la Tarea de descripción de una fotografía de la Prueba de Expresión e Interacción Oral del DELE B1 como en la del B2. Sin embargo, como el objetivo es trabajar el pensamiento divergente, también se puede utilizar este recurso en los niveles $\mathrm{C}$ ya que permitirá ahondar en temas polémicos, problemáticos y de actualidad.

\section{1 Trabajamos la variante americana}

El Instituto Cervantes introduce de forma progresiva la variante americana a partir del B1 en la Prueba de Comprensión Auditiva y de Lectura hasta llegar a encontrar muestras en la Prueba de Interacción Oral del DELE C2. Tras esta consideración, si seleccionamos cómics de Latinoamérica provocaremos además que el estudiante se familiarice con el vocabulario, así como con ciertas expresiones o modismos, propios de América Latina.

¿Cómo podemos trabajar con este recurso? Una vez presentado al autor o autora de la ilustración y a los personajes que aparecen en la tira, les mostramos a los estudiantes las viñetas con los bocadillos en blanco. 


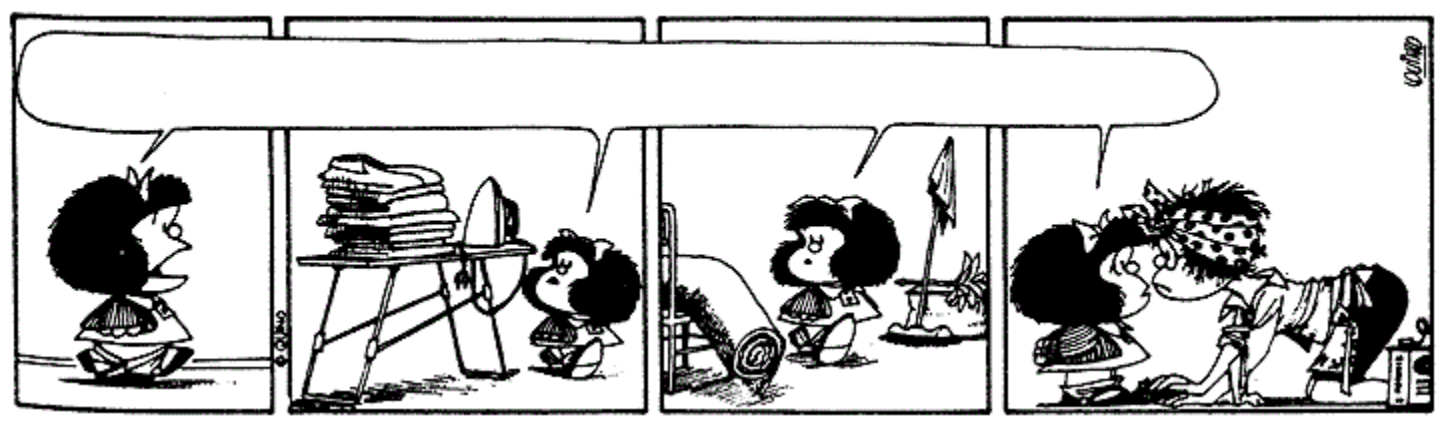

Imagen 1. Mafalda, Quino, 1991

A continuación, se analiza y se describe lo observado siguiendo la guía orientativa explicada en el punto anterior. Sobre todo, el profesor debe guiar esta práctica incidiendo en que las hipótesis creadas partan de las acciones que observan (como, en este caso, fregar, barrer, planchar, etc.).

En segundo lugar, se debatirá sobre cuál puede ser el tema que el ilustrador pretende criticar en la tira. Una vez terminada esta tarea, procedemos a darles el cómic con el texto, en el que lo primero que les llamará la atención será el voseo, así como la conjugación de los verbos y se dará una breve explicación gramatical sobre el uso del vos y de la diferente conjugación frente al español peninsular que esta forma conlleva en algunas zonas de América Latina. Tras ello, nos centraremos en analizar el contenido a través del texto y a debatir sobre el mensaje que detectamos ahora. Un ejemplo en el cómic citado es la problemática de género o la igualdad entre hombres y mujeres.

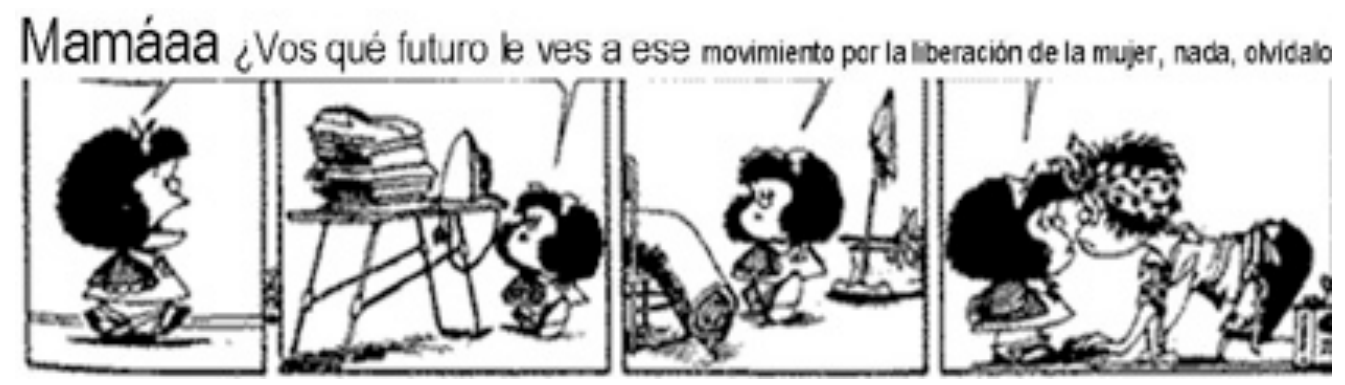

Imagen 2. Mafalda, Quino, 1991

Tras este debate abierto en el que los estudiantes se han posicionado y expresado libremente sus opiniones, procedemos a mostrarles una imagen del DELE que, sin lugar a dudas, guarde similitud con el cómic que se trabajó previamente. 


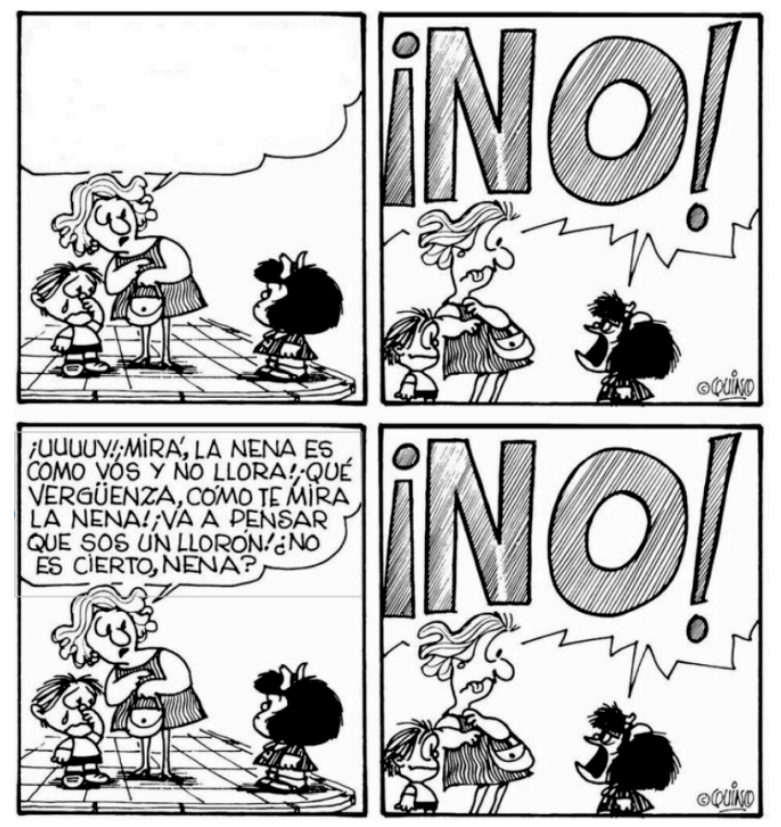

Imagen 3. Mafalda, Quino, 1991

De esta forma, el aprendiente descubrirá que posee un mayor bagaje, así como un amplio abanico de respuestas más allá de aquello que piensan que el entrevistador quiere oír.

Por otro lado, si solo nos queremos centrar en el vocabulario propio de América Latina, podemos emplear las tiras cómicas sin eliminar el texto, utilizando el procedimiento anterior y centrándonos primero en el vocabulario y la conjugación verbal, para trabajar posteriormente la reflexión y el debate abierto.

Enlazar el trabajo realizado con los cómics a la lectura de artículos periodísticos de actualidad, es otro de los puntos que también permite la explotación de este recurso.

En este sentido, el docente podría reforzar la Prueba de Comprensión Lectora (adquiriendo a su vez léxico útil para la Prueba de Comprensión Auditiva) de todos los niveles, al ofrecer al estudiante textos que trabajen una temática similar a la planteada en las viñetas. Un ejemplo serían las siguientes viñetas de Sendra y Liniers, que promueven la reflexión en torno a los artículos «Veinte años de cárcel por falsificar las notas... de sus alumnos» (ABC, 2015) o «Desafío a la diplomacia: ¿Qué hay detrás del intimidatorio apretón de manos de Donald Trump?» (Tosas, 2017) generando un debate en torno la ética y al lenguaje no verbal. 

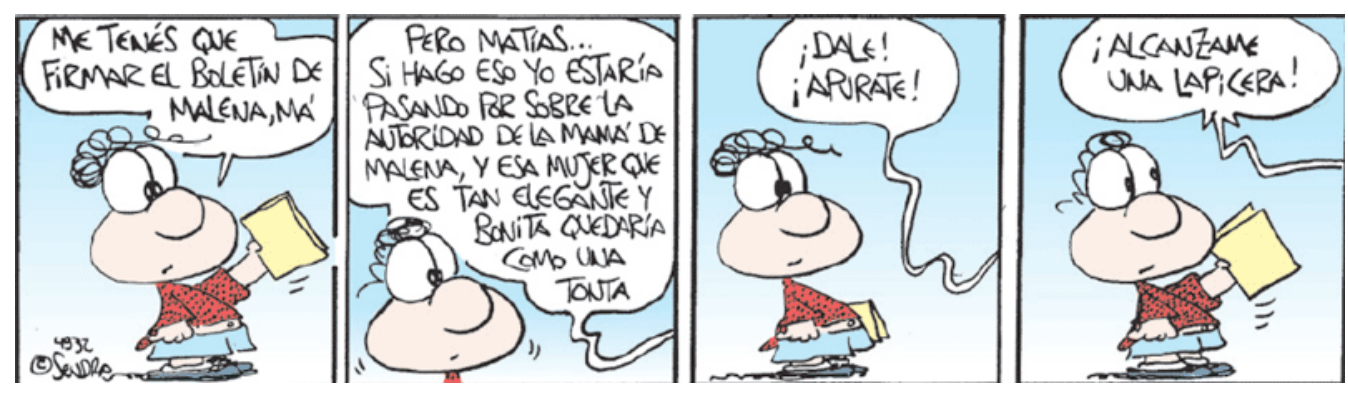

Imagen 4. Yo, Matías, Sendra, 2005
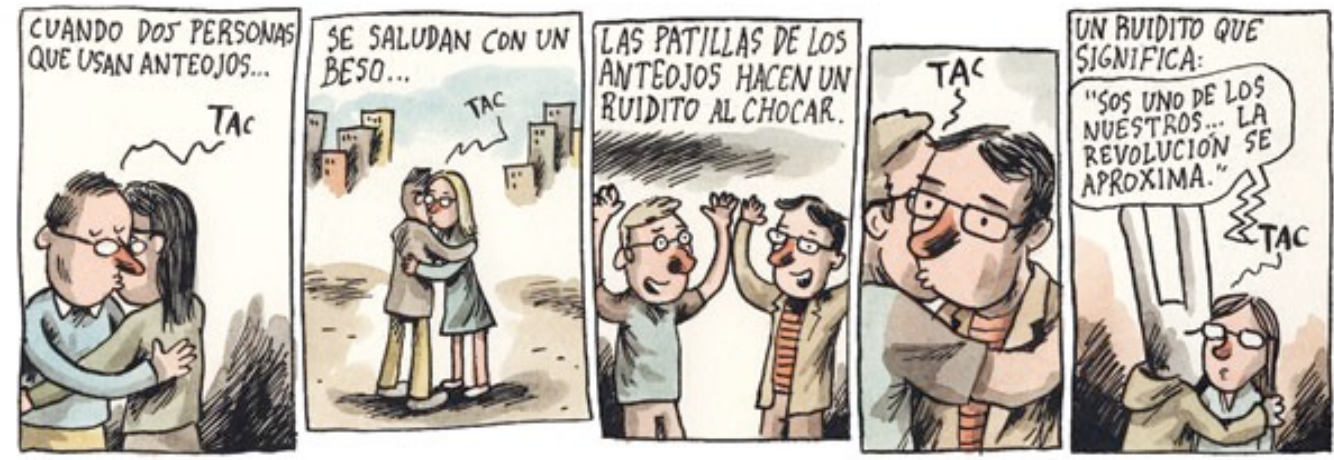

Imagen 5. Liniers, 2015

\section{El rap: un paso más en el uso de las canciones como recurso didáctico}

Como señalan María Luisa Coronado y Javier García (1990:1-2), la canción es una herramienta útil para utilizar en la clase de ELE por diversas razones: es una buena forma de relajarse y crear un ambiente distendido, estimula a los estudiantes, se obtiene una mayor fluidez, es una manera diferente de acercarse a ciertos aspectos de la cultura española y, notoriamente, es una muestra hecha por y para hispanohablantes.

El uso del rap viene determinado por unas características concretas que lo hacen idóneo para los objetivos que nos proponemos, es decir, la práctica de la pronunciación, la entonación, el lenguaje no verbal y la adquisición y consolidación de léxico, aspectos muy importantes en la Prueba de Expresión e Interacción Oral del DELE, en este caso del nivel B2.

Por un lado, tal y como indica Francisco Jiménez, al ser la lengua su herramienta y producto principal, nos encontramos en sus letras reflexiones gramaticales, figuras literarias y retóricas y, una de las características que más nos concierne, un «carácter recitado sobre una base rítmica constante» (2012: 167). Así pues, podemos decir que la complejidad de este estilo musical supone un gran interés en el aprendizaje y práctica del español y concretamente, en los aspectos que queremos trabajar.

Por otro lado, cabe destacar que estamos ante un estilo musical muy expresivo, por lo que 
se puede enfatizar su representación dentro del marco teatral y esta será precisamente la clave para trabajar con algunas de las inteligencias múltiples de Gardner (Vargas Hernández, 2004: 97-98) a saber la corporal-kinestésica, la musical, la interpersonal y la lingüística. La forma en la que estimulamos este componente dramático y a su vez, creamos un ambiente relajado, es mediante el uso de complementos de vestuario, tales como gafas de sol, gorras y herramientas diversas como tambores.

\title{
4.1 ¿Por qué Boogaloo de Kase O?
}

La canción sobre la que se asienta la actividad es Boogaloo (2011) del artista Kase O, ya que esta permite trabajar los aspectos mencionados, aunque no necesariamente de forma conjunta. Es decir, el material y los procedimientos se pueden adaptar tanto al nivel, como a las características del grupo.

\author{
Pon atención \\ Quédate con mi canción. Fiestón \\ de estilos en mi habitación, aquí es don- \\ de quedo con la inspiración, si se escon- \\ de sube la iluminación. Copón, pon \\ que rimo lo que pienso con precisión. \\ Nada se resiste a mi seducción. \\ Sexy es hoy mi exhibición. \\ Exijo Martini como Paul Desmond.
}

(Kase O, 2011)

Respecto a la pronunciación, en el texto encontramos las grafías más problemáticas para los extranjeros, por ejemplo, la «g

En cuanto al lenguaje no verbal, podemos apreciar diferentes palabras que pueden ir acompañadas por su gesto correspondiente: aquí, pienso, etc. Cabe destacar que algunas como seducción dan pie a una interpretación más abierta, por lo que el estudiante puede personalizar su actuación. En lo que se refiere al léxico, se distingue la presencia del verbo quedar (con) y sus significados. Además, suele ser frecuente tanto su uso como su confusión con quedarse (en). Por último y en relación a la entonación, el ritmo de la canción coincide con las sílabas tónicas de las últimas palabras de cada verso, las cuales en su mayoría son agudas. Así pues, se incide y se asimila fácilmente la sílaba tónica mediante la música y la palmada o golpe con el que el estudiante la acompaña, practicando como se ha mencionado anteriormente, la inteligencia musical y corporal-kinestésica.

\subsection{Procedimiento}

Esta actividad está pensada para un máximo de diez personas, aunque se puede adaptar 
dividiendo a los estudiantes en grupos más pequeños. Para empezar, se llevará a cabo la primera lectura y la explicación del léxico, así como otros recursos lingüísticos como la metáfora. A continuación, el profesor incidirá en el léxico con los fonemas más problemáticos, de forma que puedan practicar aquellos que les sean más difíciles de pronunciar. Seguidamente, se reflexionará sobre las palabras susceptibles de ser acompañadas con un gesto y se explicarán aquellos que desconozcan. Tras la familiarización con el texto y la primera audición, se entregarán las instrucciones en las que se indicará que todos deberán cantar siguiendo la melodía original, aunque con posibles variaciones en su velocidad. Además, la mitad de la clase se encargará de realizar los gestos relacionados con la letra, mientras que la otra marcará el ritmo y la sílaba tónica con un golpe o palmada. Entonces, se les proporcionará tiempo para realizar una nueva audición, si fuera necesario, y para practicar su actuación, la cual podrán acompañar de complementos de vestuario que se les facilitará. Finalmente, los alumnos podrán disfrutar realizando su actuación.

\section{3 ¿Cómo contribuye esta tarea a la preparación del DELE?}

La dramatización favorece la comunicación no verbal y, por lo tanto, beneficia la calificación holística de la Prueba de Expresión e Interacción Oral. Asimismo, con el léxico aprendido se mejora el Alcance y con la práctica de la pronunciación y de la entonación de las palabras se mejora la Fluidez, aspectos pertenecientes a la calificación analítica.

\section{Conclusiones finales}

Podemos afirmar que las propuestas aquí presentadas utilizan tres recursos de diversa índole como lo son el vídeo, la imagen y la música y que facilitan, sin lugar a dudas, el aprendizaje de los estudiantes. La estimulación a través de diferentes materiales aporta una perspectiva cognitiva diferente $\mathrm{y}$, además, permite crear una atmósfera serena en los días previos al examen.

El enfoque que ofrecemos va más allá de la forma canónica o tradicional de prepararlos exámenes del DELE realizando exámenes de manera consecutiva. Sin embargo, no nos estamos alejando mucho, puesto que algunos de los problemas que presentan los aprendientes no están relacionados directamente con la adquisición de una segunda lengua, sino que también están presentes en la suya o tienen relación con componentes sociales y culturales. Así pues, con el vídeo, se trabaja la capacidad de formular hipótesis a partir de mínima información y, seguidamente, de dotarla de coherencia. Mediante el cómic, se ejercita el pensamiento divergente y se fomenta el pensamiento crítico mediante el debate y, por último, con el rap teatralizado, se trabaja la inteligencia musical, corporal-kinestésica y la intrapersonal con el fin de desarrollar el lenguaje no verbal, que se considera una forma de comunicación muy relevante en nuestra cultura y prácticamente menos patente en otras.

Pero, ¿qué trabajamos directamente del DELE? En relación directa con los contenidos y destrezas que se exigen en estas pruebas, observamos el resultado de estas prácticas en la mejora de la Comprensión e Interacción Oral y, particularmente en el uso del cómic, en la 
de Comprensión Lectora y Auditiva.

Por un lado, centrándonos en el aspecto oral, con cualquiera de los tres recursos se verán beneficiados los apartados de Fluidez y Corrección, la Coherencia concretamente con el empleo del cómic y el vídeo y, finalmente, el Alcance con las viñetas y el rap.

Por otro lado, y en relación a la Comprensión Lectora y Auditiva se trabajará la morfología y el léxico propios de Latinoamérica mediante viñetas. No podemos olvidar que esta actividad se utilizará a su vez para fomentar el interés hacia temas de actualidad.

En definitiva, la combinación de modelos de examen con actividades de diversa naturaleza en la preparación del DELE es provechosa a nivel académico y, no menos importante, a nivel anímico, dado que es un factor que influye en la confianza del estudiante y, por consiguiente, en la óptima realización del examen.

\section{Bibliografía}

ABC (15/04/2015). Veinte años de cárcel por falsificar las notas... de sus alumnos. Recuperado de: http://www.abc.es/internacional/20150415/abci-profesora-carcel-subirnotas-201504151927.html

Coronado, L., García, J. (1990). «De cómo usar canciones en el aula». II Congreso Nacional de la ASELE. Español para Extranjeros: Didáctica e Investigación. Recuperado de: http://cvc.cervantes.es/ensenanza/biblioteca_ele/asele/asele_ii.htm

JiMÉNEZ, C. (2012). «El rap español en el ámbito de los discursos de especialidad». Pragmalingüística, vol. 24, (20), 162-184.

KASE O. (2011). «Boogaloo A.K.A Tarántula».Jazz Magnetism. [CD] España: Rap solo

LINIERS (2015). Macanudo, Madrid: PenguinRandomHouse.

QUINO (1991). 10 años con Mafalda, Buenos Aires: Ediciones de la Flor SRL.

Sendra, Fernando (2005). Yo Matías, Madrid: Losalta.

Shinzo, Abe [Prime Minister's Office of Japan] (7 de noviembre de 2013). «Japanese Prime Minister's speech: The New York Stock Exchange» [Archivo de video]. Recuperado el 02/05/2017 de: https://youtu.be/4XySzrJjVMc

SHINZO, Abe [Prime Minister's Office of Japan] (7 de noviembre de 2013). «Japanese Prime Minister's Speech: The 68th session of the UN General Assembly» [Archivo de video]. Recuperado el 02/05/2017 de: https://youtu.be/C8ElWlzslO0

Oltra, Mónica (1 de Julio de 2016). «Mónica Oltra: "Tinc molt de respecte pels votants del PP"» [Archivo de video]. Recuperado el 02/05/2017 de: https://youtu.be/iglGyHrhuYw

Oltra, Mónica (30 de mayo de 2016). «Mónica Oltra: "Bonig, el que li molesta és que es parle de les mordidas"» [Archivo de video]. Recuperado el 02/05/2017 de: https://youtu.be/1_n4OshFzAE 
TosAs, Gina (16/02/2017). ¿Qué hay detrás del intimidatorio apretón de manos de Donald Trump?, La Vanguardia, Recuperado de: http://www.lavanguardia.com/internacional/20170216/4256351790/donald-trump-apretonmanos.html

VARgas HernándeZ, A. S. (2004). «Antes y después de las inteligencias múltiples» Revista Electrónica Educare, vol. 21, (7), 91-104. 